\section{Epidemiology and Infection}

cambridge.org/hyg

\section{Original Paper}

Cite this article: Smith RP, Andres V, Cheney TE, Martelli F, Gosling R, Marier E, Rabie A, Gilson D, Davies RH (2018). How do pig farms maintain low Salmonella prevalence: a casecontrol study. Epidemiology and Infection 146, 1909-1915. https://doi.org/10.1017/ S0950268818002248

Received: 26 June 2017

Revised: 13 July 2018

Accepted: 19 July 2018

First published online: 13 August 2018

Key words:

Pig; risk factor; Salmonella; supplier

Author for correspondence:

R. P. Smith, E-mail: Richard.p.Smith@apha.gsi. gov.uk

\title{
How do pig farms maintain low Salmonella prevalence: a case-control study
}

R. P. Smith ${ }^{1}$, V. Andres ${ }^{1}$, T. E. Cheney ${ }^{1}$, F. Martelli ${ }^{1}$, R. Gosling ${ }^{1}$, E. Marier ${ }^{2}$, A. Rabie ${ }^{1}$, D. Gilson ${ }^{1}$ and R. H. Davies ${ }^{1}$

${ }^{1}$ Animal and Plant Health Agency, Woodham Lane, New Haw, Addlestone, Surrey KT15 3NB, UK and ${ }^{2}$ Veterinary Medicine Directorate, Woodham Lane, New Haw, Addlestone, Surrey KT15 3LS, UK

\begin{abstract}
Salmonella prevalence in UK pigs is amongst the highest in Europe, highlighting the need to investigate pig farms which have managed to maintain a low Salmonella seroprevalence. A total of 19 pig farms that had a consistently low $(<10 \%)$ seroprevalence over 4 years (named Platinum farms) were compared against 38 randomly selected Control farms, chosen to match the same distribution of production types and geographical distribution of the Platinum farms. Each farm was visited and floor faeces and environmental samples were collected. It was shown that Control farms had a significantly higher median percentage of pooled faecal samples positive for Salmonella compared with the Platinum farms $(12.1 \%$ and $0.4 \%$ for pooled faecal samples, respectively) and were more likely to have serovars of public health importance detected ( $S$. Typhimurium/ monophasic variants or $S$. Enteritidis). Considering the comprehensive on-farm sampling, the identification of farms negative for Salmonella, along with the identification of those that had maintained low prevalence over a long period is important. The risk factor analyses identified pelleted feed, feed deliveries crossing farm perimeter and regular antibiotic use as associated with being a Control farm. Performance data indicated that Platinum farms were performing better for slaughter live weight than Controls. Limited assessments of available pig movement records suggested that the source of pigs was not key to Platinum status, but further study would be needed to confirm this finding. These results emphasise that maintaining very low prevalence on UK farms is achievable.
\end{abstract}

\section{Introduction}

The global public health impact of non-typhoidal Salmonella was estimated to be 93.8 million illnesses per year, of which approximately 80.3 million were foodborne [1]. More than 80000 cases are reported in the European Union (EU) each year, with an estimated overall economic burden of approximately 3 billion Euros per year [2]. Although a recent decrease in the number of human Salmonella cases in the EU has been observed, salmonellosis is consistently the second most frequently reported zoonosis $[2,3]$. This pathogen remains the most common causative agent responsible for foodborne outbreaks and pig meat was the most common food vehicle for Salmonella Typhimurium outbreaks in 2013 and 2014 [3].

Salmonella Enteritidis and S. Typhimurium are the most commonly reported serovars associated with human Salmonella cases and in 2014 accounted for $44.4 \%$ and $17.4 \%$ of reported cases, respectively [3]. These zoonotic Salmonella are carried by a large variety of farmed livestock, avian species and mammalian wildlife, as well as a number of common and exotic pet animals such as dogs and reptiles $[4,5,6]$. Pigs are infected by numerous serovars of Salmonella, with S. Typhimurium and its two monophasic variants (S. 4, 5, 12:i:- and $S$. 4,12:i:-), S. Derby and $S$. Bovismorbificans being the most common in British finisher pigs [7]. Salmonella infection in weaned pigs can cause fever, enteritis, septicaemia and mortality, as well as a reduction in growth rate in apparently unaffected pigs [8]. An EU baseline survey detected a high prevalence of $21.2 \%$ in lymph nodes of healthy UK slaughter pigs $[9,10]$, while a prevalence of $30.5 \%$ in caecal samples from slaughtered pigs was detected by a prevalence study in 2013 [7] which highlights the need to identify effective Salmonella control strategies. However, most infections are subclinical and widespread amongst multiple epidemiological groups and production stages, making the detection of infected pigs on the farm and subsequent control, difficult and costly. To help monitor pig Salmonella, a National programme was introduced in 2002 to estimate the burden of Salmonella in pigs sent to slaughter by testing meat juice samples for antibodies against Group B and C1 Salmonella [11], although sample testing was subsequently suspended in 2012 to refocus the scheme. A positive serological result was assumed to represent prior infection and not necessarily a current infection, but studies have shown that serological results provide an appropriate proxy for herd prevalence [12]. 
Previous studies of factors related to Salmonella infection in pigs have identified a large range of risk factors. The factors relating to on-farm control can be grouped into a number of key areas: internal and external biosecurity, feed and water, treatments and co-infection. External biosecurity relates to preventing Salmonella from entering the farm, whereas internal biosecurity refers to management to restrict Salmonella transmitting within the farm. Previous studies have shown that farms that use homebred replacement pigs, or utilise few sources of pigs, are at lower risk than those that use many sources [13]. This aspect also includes the quarantine of incoming stock and the control of wildlife, which also present a risk of introducing and circulating Salmonella on a farm $[14,15]$. To limit the transmission from infected pigs or the contaminated environment to uninfected pigs, effective cleaning and disinfection between batches of pigs have been shown to be useful, as has the use of slatted flooring which separates most contaminated waste from the pig's vicinity and allows each pen to be managed independently $[16,17]$.

The use of acidified feed or water, or fermented liquid feed, which help create an acidic gut environment, have been shown to reduce Salmonella. Salmonella growth and survival are inhibited by an acidic environment and an acidic environment favours other bacteria, allowing them to out-compete Salmonella for resources $[18,19,20]$. Additionally, the composition of feed: pellets or meal; particle size of the feed used to make pellets; and proportions of wheat and barley, have all been shown to be associated with Salmonella infection in pigs [13, 21, 22]. The use of broadspectrum antibiotics disrupts the normal protective gut flora and has been associated with increased Salmonella infection [19, 23]. Conversely, the uses of probiotics and prebiotics may help reduce Salmonella prevalence by promoting protective gut flora [24, 25]. Finally, co-infection with a number of other pathogens, such as those causing enzootic pneumonia, migratory Ascaris suum infection ('milk spot') and Porcine Reproductive and Respiratory Syndrome, have been shown to be associated with increased Salmonella prevalence, possibly due to interference with the host's immune response [19, 26, 27].

Previous research has concentrated on studying Salmonella on high prevalence pig farms, or within the general farm population and the factors that have led to farms maintaining a low prevalence have not been studied in detail. For example, Gotter [28] compared farms classified into high and low seroprevalence groups by a single year of slaughterhouse surveillance to determine risk factors. The aim of this project was to identify factors associated with maintaining a low Salmonella seroprevalence over a 4-year period using a case-control design. The study also utilised extensive on-farm sampling and bacteriological testing to further explore the difference in presence and diversity of Salmonella strains on the farm. To investigate whether any apparent difference between the farms could be attributed to differences in the use of pig suppliers, incoming pig movements were also assessed to complete this comprehensive comparison between the two groups of farms.

\section{Material and methods}

\section{Farm selection}

A selection of 50 pig farms with consistently low seroprevalence $(<10 \%$ of pigs a year seropositive at slaughter) was identified through the use of the last 4 complete years of the UK serological surveillance scheme data (2008-2011) and hereinafter referred to as Platinum farms. To account for changes to prevalence from that time point to the sampling for this study (July 2013-May 2014), finisher pigs from the Platinum farms that agreed to join the study were sampled at slaughter and any farms found to have sample results that would have removed their Platinum status $(>10 \%$ prevalence of serovars detected by the meat juice ELISA from 60 samples) were omitted from the study. A total of 19 Platinum were eligible to join the study. Three farms from the initial 50 were sampled and found to have lost their Platinum status and a study of these farms has been published elsewhere [29].

For each Platinum farm, two Control farms were randomly selected from a list of national pig holdings held at Animal and Plant Health Agency to provide a comparable study population from the wider pig industry. The 50 low seroprevalence farms that were originally selected to recruit Platinum farms were omitted from being selected as Control farms. The Controls were not individually matched to the Platinum farms but were selected to produce the same distribution of production types (indoor/outdoor; breeder/finisher) and geographical distribution as the Platinum farms, to control for expected differences between the Platinum farms and the background population. Only 26 of the 38 recruited Control farms could be linked by farm identifiers to seroprevalence data over the same period and the results indicated an average seroprevalence of $28 \%$. Although a proportion (between $11 \%$ and 35\%) of these achieved a seroprevalence below $10 \%$ in a single year, none consistently met this target.

Due to the small number of farms with consistently low seroprevalence, comparisons between the Platinum and Control farms would detect an Odds Ratio (OR) of 5.5 with $95 \%$ confidence and $80 \%$ power, assuming a prevalence of $20 \%$ in the Control population. The sample size would assist with detecting strong interventions likely to provide cost-benefit at the farm-level.

\section{Sample collection and laboratory testing}

Each enrolled farm was visited once during which pooled and individual floor faeces and environmental samples were collected from pigs and their surroundings to provide bacteriological evidence for the previous serological status of each farm and compare the presence and diversity of Salmonella strains on Platinum and Control farms.

Each building containing pigs was sampled, with up to 20 pooled faeces samples collected from each building. Each sample was approximately $25 \mathrm{~g}$ and was collected using a sterile hand held gauze swabs. Generally, a single pooled sample was taken from one pen out of every two or three pens to produce a representative selection from the building. However, if there were less than 20 pens present in a building then all pens were sampled. If more than 50 pigs were present in a pen, then two or more pooled samples were collected to provide adequate representation within the 20 samples allotted for that building.

Where possible, 60 (10 samples from six pens) samples of individual fresh faecal droppings were collected from finishing pigs' pens (or growers, if too few finisher pens were available). Environmental samples (up to 70 per farm) collected using sterile hand gauze swabs were gathered from boot dips, walkways, pooled water, feed, drinkers, feeders, farm effluent, pig handling equipment, vehicle foot wells, protective clothing, scrapers and wildlife faeces. For surfaces, an area of approximately $1 \mathrm{~m}^{2}$ was swabbed. The selection of the environmental samples was determined by which sources were present and by the decision of the experienced 
member of sampling staff of which sources might have had the most influence on the pigs.

The testing method used was a modification of the ISO 6579:2002 (Annex D) Salmonella isolation method in which only one plating medium (Rambach agar) is used [30]. Briefly, the samples were placed directly into $225 \mathrm{ml}$ of the pre-enrichment culture media (Buffered Peptone Water-BPW; Merck 10.07228.0500) at the farm. All were incubated at $37 \pm$ $1{ }^{\circ} \mathrm{C}$ for $16-20 \mathrm{~h}$ and then $0.1 \mathrm{ml}$ of broth was inoculated onto modified semi-solid Rappaport-Vassiliadis enrichment agar (MSRV; Mast DM440D, with addition of $1 \mathrm{mg} / \mathrm{ml}$ of novobiocin, Sigma N1628) and incubated at $41.5 \pm 1{ }^{\circ} \mathrm{C}$ for $24 \pm 3 \mathrm{~h}$. Growth on the MSRV agar was sub-cultured onto Rambach agar (Merck 1.07500.0002) and incubated at $37 \pm 1{ }^{\circ} \mathrm{C}$ for $24 \pm 3 \mathrm{~h}$. Slide agglutination tests on suspect colonies were carried out to confirm Salmonella presence. All positive Salmonella isolates were serotyped according to the White-Kauffmann-Le Minor scheme [31].

\section{Data collection}

At each farm visit, a questionnaire was completed to provide explanatory data for risk factor analysis (please see supplementary material). Detailed information related to business characteristics, housing systems, animal sourcing, husbandry, management and feeding practices, as well as health and pig performance, were collected. To further explore the potential differences between the Platinum and Control farms, an assessment of their pig suppliers was completed. Routinely collected pig movement data (eAML2, http://www.eaml2.org.uk/ami/home.eb) was gathered from a time period 12 months prior to their farm visit.

\section{Data analysis}

Statistical analyses were completed in Stata 12 (Statacorp, College Station, Texas, USA). To compare bacteriological sample results from the Platinum and Control farms, a mixed-effects logistic regression was completed (command xtmelogit), with Farm ID included as a random effect to account for the clustering of sample results at the farm-level. Comparisons were made to ascertain significant associations between the farm status and the results from pooled and individual faecal samples and each type of environmental sample, which were used as the outcomes in these univariable sample-level models.

For the risk factor analysis, to identify factors potentially associated with persistent low Salmonella prevalence, a farm-level logistic regression model was completed in, with the outcome variable being whether a farm was classified as Platinum (1) or Control (0) and the questionnaire responses used as independent variables. Continuous variables (such as total herd size) were plotted as histograms to determine whether they approximated normality and were tested in the model for a linear relationship with the outcome. Variables that were either considered nonnormal or did not have an apparent linear relationship were reanalysed as a categorical variable, with the categories typically representing quartiles or quintiles. Due to a large number of independent variables, an initial univariable screening stage was used to assess all independent variables, with those with a $P$-value $>0.3$ omitted from further analysis. A forward stepwise method was then used to select from the selected variables to determine which should enter the model at each step. Likelihood Ratio tests were used to compare models to determine whether an included variable significantly improved the model. The final multivariable model was fitted from variables retained in the model that had a Likelihood Ratio $P$-value $<0.05$. A collinearity matrix (command pwcorr) was produced to identify variables strongly collinear ( $>0.7)$ with variables entering the final model. Any variable found to be collinear with a variable already existing in the model was omitted. Potential confounding between variables was also investigated by monitoring whether the addition of variables at each step inflated the OR of the variables retained in the model. The overall model fit was assessed using a Likelihood Ratio $\chi^{2}$ test. No biologically plausible interactions were expected between the independent variables and so no interaction terms were tested.

Data from the eAML2 system was used to compare the use of pig suppliers between the Platinum and Control farms. Only those farms that could perfectly match farm identifiers (County Parish Holding number and Postcode) from the farm questionnaire to the eAML2 system were used for the descriptive analysis. For those farms which successfully linked to pig movement records, the dataset was described to identify suppliers that provided pigs to both Platinum and Control farms and the number and proportion of pigs supplied by each supplier to each farm in the 12-month period. Comparisons were also made of sample results from Platinum and Control farms found to have used the same supplier.

\section{Results}

The 19 recruited Platinum farms and 38 Control farms were both $16 \%$ specialist finisher farms and $84 \%$ farrow-to-finish farms, with $37 \%$ from the North of England (Yorkshire, Lancashire, Durham and Lincolnshire), 37\% from the South (Hampshire, Wiltshire, Gloucestershire, Cornwall, Devon and Somerset) and $26 \%$ from the Midlands (Warwickshire, Leicestershire, Nottinghamshire, Bedfordshire, Shropshire, Oxfordshire and Staffordshire). One Platinum farm and two Controls farrowed outdoors. The farms ranged in size from 150 pigs to 9000 pigs (mean 2900), with more variability in size in the Controls (mean 3,040, standard deviation 2391) than in the Platinum farms (mean 2,691, S.D. 1871).

A total of 11452 samples were collected from the 57 farms, with 9746 of these comprising pen floor faeces (Table 1). Overall, a mean of 165 and 138 pooled faecal or environmental samples were collected from each of the 19 Platinum and 38 Control farms, respectively. For the individual faecal samples from grower/finisher pigs, 57 on average were collected from Platinum farms and 54 from Controls. The highest proportion of Salmonella-positive samples from a summary of results from all of the farms was from waste handling equipment $(37.1 \%)$, vehicles $(37.1 \%)$, run-off and pooled water from puddles on the farm (29.1\%) (Table 1).

On individual farms, the average of the percentage of positive pooled faecal samples from the Platinum farms was $4.3 \%$ (median $0.4 \%$, range $0.0 \%-25.8 \%$ ) with $9 / 19$ farms (47.4\%) negative for Salmonella. The average percentage of positive individual faecal samples from the Platinum farms was $0.1 \%$ (median $0.0 \%$, $0.0 \%-1.7 \%$ ) with only a single farm being Salmonella-positive. At farm-level, the Control farms had an average percentage of positive pooled samples of $19.4 \%$ (median $12.1 \%, 0.0 \%-52.4 \%$ ) and with $3 / 38$ Control farms (21\%) having no Salmonella detected. The average percentage of positives from the Control's individual samples was $6.7 \%$ (median $0.0 \%, 0.0 \%-61.7 \%$ ). 
Table 1. Summary of Salmonella results by sample type or location of environmental swabbing and mixed-effects model outputs comparing sample results from the two farm types (Farm ID used as a random effect)

\begin{tabular}{|c|c|c|c|c|c|c|c|c|}
\hline \multirow[b]{2}{*}{ Sample type } & \multicolumn{3}{|c|}{ Platinum farms } & \multicolumn{3}{|c|}{ Control farms } & \multicolumn{2}{|c|}{ Model result } \\
\hline & Positive & Negative & \%pos. & Positive & Negative & $\%$ pos. & Odds Ratio & $P$-value \\
\hline Pen floor faeces from pigs & 68 & 3489 & 1.9 & 924 & 5265 & 14.9 & 0.07 & $<0.01$ \\
\hline Pooled faeces samples & 67 & 2405 & 2.7 & 786 & 3402 & 18.8 & 0.07 & $<0.01$ \\
\hline Individual faeces samples & 1 & 1084 & 0.1 & 138 & 1863 & 6.9 & 0.01 & 0.02 \\
\hline Sick Pen & 1 & 43 & 2.3 & 20 & 27 & 42.6 & 0.01 & 0.04 \\
\hline Boot Dip & 0 & 22 & 0.0 & 1 & 38 & 2.6 & $\mathrm{n} / \mathrm{a}$ & \\
\hline$C \& D^{a}$ pen & 0 & 68 & 0.0 & 9 & 77 & 10.5 & $\mathrm{n} / \mathrm{a}$ & \\
\hline Pig handling equipment & 0 & 35 & 0.0 & 13 & 31 & 29.5 & $\mathrm{n} / \mathrm{a}$ & \\
\hline Walkways & 10 & 143 & 6.5 & 71 & 138 & 34.0 & 0.11 & $<0.01$ \\
\hline Wild bird faeces & 3 & 19 & 13.6 & 15 & 37 & 28.8 & 0.55 & 0.34 \\
\hline Rodent faeces/carcases & 0 & 41 & 0.0 & 4 & 61 & 6.2 & $\mathrm{n} / \mathrm{a}$ & \\
\hline Pet/wild mammal faeces & 0 & 3 & 0.0 & 0 & 1 & 0.0 & $\mathrm{n} / \mathrm{a}$ & \\
\hline Other farm species & 0 & 8 & 0.0 & 4 & 24 & 14.3 & $\mathrm{n} / \mathrm{a}$ & \\
\hline Water sources & 1 & 31 & 3.1 & 0 & 59 & 0.0 & $\mathrm{n} / \mathrm{a}$ & \\
\hline Feed sources & 1 & 75 & 1.3 & 7 & 95 & 6.9 & 0.17 & 0.13 \\
\hline Run Off/pooled water & 5 & 74 & 6.3 & 62 & 89 & 41.1 & 0.04 & $<0.01$ \\
\hline Waste handling & 6 & 33 & 15.4 & 40 & 45 & 47.1 & 0.10 & 0.01 \\
\hline Vehicles & 5 & 33 & 13.2 & 38 & 40 & 48.7 & 0.17 & 0.02 \\
\hline
\end{tabular}

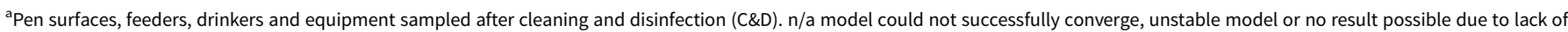
positive values.

Comparisons of the sample results from all the farms of each of the two farm types, adjusting for clustering of results at farmlevel, showed that Platinum farms were significantly $(P<0.05)$ associated with a lower odds of both pooled faeces and individual faeces being Salmonella positive (Table 1). The difference in overall percentage of positive samples between the Platinum and Control farms was also reflected in the environmental samples (Platinum $=4.8 \%$; Controls $=27.2 \%$ ), with Control farms significantly more likely to have positive samples in individual comparisons between samples from vehicles, waste handling equipment, run-off and pooled water, walkways and sick pens (Table 1). No significant association was detected for wild bird faeces and feed sources, whereas the models for the other environmental samples failed to successfully converge or produce modelling errors.

The most common serovar of major public health importance on Control farms was $S$. Typhimurium (398 pooled sample isolates on 13 farms and 73 individual samples on eight farms), followed by either monophasic $S$. Typhimurium strain (S. 4,12:i:- or S. 4,5,12:i:-, 166 isolates on 12 farms) and Salmonella Enteritidis (3 isolates on 2 farms), whereas only two Platinum farms had monophasic S. Typhimurium (S. 4,12:i:-, 15 isolates). The most detected serovar on Platinum farms was $S$. Reading (49 isolates) which was found in pooled samples from two farms. Only one serovar was detected from individual samples from the Platinum farms; a single $S$. Agama isolate, which is likely to represent transient contamination of feed by badgers [32].

The results from the risk factor analysis showed that the variable related to feed lorries having access to the farm was strongly collinear with live pig transport entering the farm perimeter
(0.72) and any vehicles entering the farm perimeter (0.84). As expected, the levels of the combined feed type variable were strongly collinear with binary variables for each of the individual feed types. The collinear variables (live pig transport, vehicles entering perimeter and individual feed types) were not included in the multivariable model as the other variables had been shown to improve the model and had been selected to be retained in the model. The final multivariable risk factor model enabled identification of explanatory variables associated with Salmonella seroprevalence status. The analysis identified three significant variables, showing that Control farms were more likely to use pelleted feed; routinely add antibiotics to feed or water; and allow feed lorries to enter beyond the farm perimeter (Table 2). No apparent confounding effect was detected between the variables retained in the model. The result of the Likelihood Ratio $\chi^{2}$ test indicated that the null hypothesis, of no effect of the independent variables on the outcome, could be rejected.

Although not retained in the farm-level model, an interesting finding was that a significant difference was detected at the univariable stage between a performance indicator (slaughter live weight) and whether a farm was a Platinum or a Control. Platinum farms had a higher slaughter live weight (mean Platinum $103.7 \mathrm{kgs}$ (quartile range 100.0-114.5), mean Control $93.8 \mathrm{kgs}(80.5-106.0), \quad P$-value $=0.029, \quad n=46)$. Controls also had a higher post-weaning mortality of weaners but the result was only approaching significance in the univariable analysis (mean Platinum 0.8, mean Control 2.1, $P$-value $=0.062, n=46$ ).

Data on the use of pig suppliers could be matched from the eAML2 system to 12 Platinum farms and 20 Control farms. No significant difference was detected in farm demographics (herd 
Table 2. Farm-level Salmonella risk factors associated with being a Platinum (low-prevalence) farm identified through multivariable analysis of 57 pig farms

\begin{tabular}{|c|c|c|c|c|c|c|}
\hline Variable & Level & $\begin{array}{l}\text { Platinum } \\
\text { farms }\end{array}$ & $\begin{array}{l}\text { Control } \\
\text { farms }\end{array}$ & $\begin{array}{l}\text { Odds } \\
\text { Ratio }\end{array}$ & $\begin{array}{l}95 \% \text { confidence } \\
\text { intervals }\end{array}$ & $P$-value \\
\hline \multirow[t]{3}{*}{ Feed type most commonly used } & Pelleted & 2 & 27 & 1.00 & - & - \\
\hline & Meal & 8 & 7 & 19.88 & $2.19-180.85$ & 0.008 \\
\hline & Liquid & 9 & 4 & 27.11 & $3.03-242.32$ & 0.003 \\
\hline \multirow{2}{*}{$\begin{array}{l}\text { Feed lorries ever enter the farm } \\
\text { perimeter }\end{array}$} & Yes & 7 & 32 & 1.00 & - & - \\
\hline & No & 12 & 6 & 10.93 & $1.78-67.00$ & 0.010 \\
\hline \multirow[t]{2}{*}{ Antibiotics regularly added to feed/water } & Yes & 5 & 26 & 1.00 & - & - \\
\hline & No & 14 & 12 & 4.84 & $0.92-25.40$ & 0.062 \\
\hline
\end{tabular}

size, herd type, farrowing system) from $\chi^{2}$ and $t$-tests between the population that could be linked to pig movements and those that could not, indicating no apparent bias in demographics. The Platinum farms used a total of 16 unique suppliers and the Controls used 29 unique suppliers, with eight suppliers sending pigs to both Platinum and Control farms. For combinations of Platinum and Control farms that shared the same supplier, the Salmonella results were descriptively compared to examine whether supply source had a strong effect on the receiving farm (Table 3). For most of the farms that shared suppliers, the percentage of positive pooled samples and serovars detected differed. To make an effective comparison, farms from which the supplier did not supply the majority of incoming pigs $(>90 \%)$ to the farms and those that only received very few pigs $(<10$ pigs $)$ from the suppliers were omitted from further analysis. Of the remaining four suppliers (suppliers 1, 2, 5 and 6), each had only one Platinum and one Control farm remaining for comparison. Platinum and Control farms linked to three of these suppliers had a similar percentage of positive samples $( \pm 5 \%)$ while the other supplier was linked to farms where the percentage differed greatly $(>50 \%)$ between the two farm populations. Only one pair of Platinum and Control farms, using the same supplier (supplier 5), had serotype results for both farms and these indicated that the same serotypes were not present in both farms.

\section{Discussion}

The results of the farm visits confirmed that the participating Platinum farms, which had a persistently low seroprevalence up until the end of 2011, had also maintained a low prevalence, as assessed by bacteriological testing when visited in 2013/14. Many of the Platinum farms were also found to be Salmonella-free, which was a surprising finding considering the comprehensive sampling of the farms, use of highly sensitive pooled faecal samples and a culture method which can identify small concentrations of Salmonella [33, 34]. However, an improvement to the study would have been a longitudinal sampling of the farms to account for any intermittent shedding of Salmonella. The results also indicated a difference in detection of serovars between Platinum farms and Controls. This was particularly evident for $S$. Typhimurium and its monophasic variants (STM), which are of major public health importance, indicating that the Platinum farms presented a lower risk to public health. The identification of farms that have been able to maintain such low prevalence in a country which has a relatively high prevalence in pigs compared with other EU Member States [9] is important and these farms could be used as exemplars for others to show that Salmonella control is achievable and sustainable.

The difference of the percentage of Salmonella-positive samples between pig faeces from the two sets of farms was also replicated in many of the environmental samples. These results and the identification of significant differences in the percentage of positives detected for specific sampled areas, such as walkways in pig buildings, suggest that the Platinum farms were more successful at reducing contamination on farm and that the higher percentage of positives in the Controls may have allowed for more effective recirculation of Salmonella between batches of pigs. However, the non-standardised and opportunistic nature of the environmental sampling may have led to biases in the comparison, as not all farms had the same sources sampled and a greater proportion of Salmonella-positive samples were detected in some sources than in others.

The Platinum farms were also found to have measurably better pig performance than the Control farms for two performance indicators, which may suggest that enhanced farm management is associated with both lower Salmonella prevalence and better pig performance. Salmonella infection is typically subclinical in pigs, other than in recently weaned piglets and so improved pig weight and lower mortality may in part result from controls that affect Salmonella, also helping to reduce infection by other porcine pathogens. This finding could be useful in encouraging farmers to apply Salmonella interventions. However, the indicators were not retained in the final model. It should be noted that slaughter weight may also reflect differences in management, with farms agreeing to send larger or smaller pigs to slaughter due to the buyer's requirements, rather than poor performance. Comparisons of other performance indicators did not show a significant difference between the groups.

At farm-level, the multivariable risk factor analysis compared the practices applied on the two groups of farms and highlighted that those using liquid and meal feed were at lower risk than those feeding pelleted feed and those regularly applying antibiotics to feed or water were at greater risk. This use of antibiotics was not reflective of clinical salmonellosis, which was present on only two farms (Controls). Use may reflect that Control farms had more clinical health conditions than the Platinum farms or were using antibiotics prophylactically to control ongoing health issues. It is unknown whether the detected association with Salmonella was due to the effect of the antibiotics themselves or co-infection with the health conditions that were being treated. However, this finding may help encourage farms to reduce 
Table 3. Pooled sample Salmonella result comparisons where Platinum and Control farms shared the same pig supplier

\begin{tabular}{|c|c|c|c|c|c|}
\hline $\begin{array}{l}\text { Supplier } \\
\text { ID }\end{array}$ & $\begin{array}{l}\text { Farm } \\
\text { type }\end{array}$ & $\begin{array}{l}\% \text { of samples } \\
\text { Salmonella positive }\end{array}$ & Salmonella serovar (no. of isolates) & $\begin{array}{l}\text { no. pigs from } \\
\text { supplier }^{\mathrm{a}}\end{array}$ & $\begin{array}{l}\% \text { of pigs from } \\
\text { supplier in a year }\end{array}$ \\
\hline 1 & $P$ & 0.0 & - & 30 & 100.0 \\
\hline 1 & C & 0.0 & - & 13 & 100.0 \\
\hline 1 & C & 33.3 & $\begin{array}{l}\text { 4,12:i:- (3), 4,5,12:i:- (4), Derby (15), London (2), } \\
\text { Newport (1), Reading (3) }\end{array}$ & 4 & 1.3 \\
\hline 2 & $\mathrm{P}$ & 0.0 & - & 2400 & 100.0 \\
\hline 2 & C & 2.8 & 4,5,12:i:- (4), Coeln (1) & 14000 & 100.0 \\
\hline 3 & $\mathrm{P}$ & 0.7 & Mbandaka (1) & 1 & 3.0 \\
\hline 3 & $\mathrm{P}$ & 0.0 & - & 3 & 100.0 \\
\hline 3 & C & 35.9 & Kedougou (46), Typhimurium (33) & 3 & 100.0 \\
\hline 3 & C & 47.2 & London (16) & 2 & 100.0 \\
\hline 3 & C & 33.3 & $\begin{array}{l}\text { 4, 12:i:- (3), 4, 5, 12:i:- (4), Derby (15), London } \\
\text { (2), Newport (1), Reading (3) }\end{array}$ & 8 & 2.7 \\
\hline 4 & $\mathrm{P}$ & 2.2 & Kedougou (1), Derby (1) & 6 & 100.0 \\
\hline 4 & C & 33.1 & 4, 12:i:- (15), Typhimurium (83) & 7 & 100.0 \\
\hline 5 & $\mathrm{P}$ & 0.7 & Mbandaka (1) & 30 & 97.0 \\
\hline 5 & C & 5.6 & $\begin{array}{l}\text { Agama (3), Ajiobo (1), Derby (4), Stourbridge } \\
\text { (1) }\end{array}$ & 130 & 100.0 \\
\hline 6 & $\mathrm{P}$ & 0.0 & - & 160 & 90.8 \\
\hline 6 & $P$ & 8.7 & 4, 12:i:- (6) & 70 & 1.3 \\
\hline 6 & C & 50.3 & $\begin{array}{l}\text { Enteritidis (1), Kedougou (2), Typhimurium } \\
\text { (69) }\end{array}$ & 60 & 100.0 \\
\hline 7 & $\mathrm{P}$ & 8.7 & 4, 12:i:- (6) & 1 & $<0.1$ \\
\hline 7 & C & 7.9 & 4, 12:i:- (17), Kedougou (10) & 6 & 0.4 \\
\hline 8 & $\mathrm{P}$ & 0.0 & - & 16 & 9.2 \\
\hline 8 & $\mathrm{C}$ & 7.9 & 4, 12:i:- (17), Kedougou (10) & 900 & 54.6 \\
\hline
\end{tabular}

P, Platinum, C, Control.

${ }^{a}$ Numbers over 20 have been rounded to two significant figures.

antibiotic use, which would support wider aims to reduce antimicrobial resistance within the industry. These have been common findings in other studies and relate to maintaining a good gut environment that is inhibitory to Salmonella and allows nonpathogenic bacteria to outcompete Salmonella for intestinal attachment sites, nutrients and to produce inhibitory bacteriocins and organic acids $[18,19,20]$. The Platinum farms were also less likely to allow feed lorries to enter the farm. This factor was collinear with other variables related to vehicle access to the farm, which may indicate that it could be a proxy for wider enhanced levels of external biosecurity, indicating these farms were more likely to be actively trying to stop pathogens entering the farm. The studied population size would only detect significantly associated variables that had an OR of 5.5 or greater with confidence, whereas risk factors with weaker associations with the outcome may have been missed.

Pig supply has often been cited as the number one cause of Salmonella prevalence [35]. However, the data collected from the study farms did show that a small number of suppliers were the major provider of pigs to both Platinum and Control farms, which suggests that the differences in percentage of positive Salmonella samples between these farms were not simply due to the use of different suppliers and shows the importance of on-farm control factors. However, it is unknown how supplier use had changed during the period in which the Platinum farms maintained low seroprevalence (2008-2011) and the results from our sampling in 2013-14 may reflect that recent changes of supplier in the Control population had resulted in a similar low percentage of positive samples detected in both Platinum and Control farms for three of the four suppliers used in the final investigation. This analysis was limited by the lack of data from all participating farms. Not all farms could be linked to pig supplier information, either because of problems with issues in the recording of farm identifiers or because these farms were closed and had no incoming pig movements.

This study was not representative of all the pig industry. As the Platinum farms were those that previously submitted finisher pigs for testing within the serological surveillance system and the Controls were selected to match the same distribution of production types and regions covered, specialist breeding farms were not included in the study. Additionally, it was noticeable that the Platinum farms were not present in East Anglia, a high pig farm density area of Great Britain, although it is expected that many of the farms in this region are breeding farms. Due to the selection bias in the study, the results cannot be generalised to these areas of the pig industry. 


\section{Conclusions}

This study has identified important differences between farms that have maintained a low prevalence and other commercial herds, encompassing differences in the presence and diversity of Salmonella and management practices on the farms. It is hoped that this information will help motivate the pig industry that the ability to produce and maintain a low Salmonella herd is achievable. Control efforts may need to cover improvements to biosecurity, feed and treatment and improved cleaning and disinfection to reduce environmental contamination, in order to tackle Salmonella and have a positive effect on food safety and overall herd productivity.

Supplementary material. The supplementary material for this article can be found at https://doi.org/10.1017/S0950268818002248

Acknowledgements. The authors would like to thank the farmers, their staff and their vets for their participation and assistance with this study. APHA staff are also thanked for their help with sampling these farms, processing the samples and data entering the information. Bhagyalakshmi Chengat is also thanked for her help in validating the model building approach. This work was supported by the Department for Environment, Food and Rural Affairs (DEFRA) (project reference OZ0344).

Conflicts of interest. None

\section{References}

1. Majowicz SE et al. (2010) International Collaboration on Enteric Disease 'Burden of Illness' studies. The global burden of nontyphoidal Salmonella gastroenteritis. Clinical Infectious Diseases 50, 882-889.

2. EFSA/ECDC (2012) The European Union Summary report on trends and sources of zoonoses, zoonotic agents and food-borne outbreaks in 2010. EFSA Journal 10, 2597.

3. EFSA (2015) The European Union Summary report on trends and sources of zoonoses, zoonotic agents and food-borne outbreaks in 2014. EFSA Journal 13, 4329.

4. Geue L and Loschner U (2002) Salmonella enterica in reptiles of German and Austrian origin. Veterinary Microbiology 84, 79-91.

5. Simpson VR (2002) Wild animals as reservoirs of infectious diseases in the UK. Veterinary Journal 163, 128-146.

6. APHA (2013) Salmonella in Livestock Production in GB. Available at https://www.gov.uk/government/publications/salmonella-in-livestock-production-in-great-britain-2013. (Accessed 10 January 2017).

7. Powell LF et al. (2016) A prevalence study of Salmonella spp., Yersinia spp., Toxoplasma gondii and porcine reproductive and respiratory syndrome virus in UK pigs at slaughter. Epidemiology and Infection 144, 1538-1549.

8. Farzan A and Friendship RM (2010) A clinical field trial to evaluate the efficacy of vaccination in controlling Salmonella infection and the association of Salmonella-shedding and weight gain in pigs. Canadian Journal of Veterinary Research 74, 258-263.

9. EFSA (2008) Report of the task force on zoonoses data collection on the analysis of the baseline survey on the prevalence of Salmonella in slaughter pigs, in the EU, 2006-2007 [1] - part A: Salmonella prevalence estimates. EFSA Journal 135, 1-111.

10. Marier EA et al. (2014) Abattoir based survey of Salmonella in finishing pigs in the United Kingdom 2006-2007. Preventive Veterinary Medicine 117, 542-553.

11. Snary EL et al. (2010) Zoonoses action plan Salmonella monitoring programme: an investigation of the sampling protocol. Journal of Food Protection 73, 488-494.

12. Sorensen LL et al. (2004) The correlation between Salmonella serology and isolation of Salmonella in Danish pigs at slaughter. Veterinary Microbiology 101, 131-141.
13. Lo Fo Wong DMA et al. (2004) Herd-level risk factors for subclinical Salmonella infection in European finishing-pig herds. Preventive Veterinary Medicine 62, 253-266.

14. Henzler DJ and Opitz HM (1992) The role of mice in the epizootiology of Salmonella Enteritidis infection on chicken layer farms. Avian Diseases 36, 625-631.

15. Muirhead S (1993) House mice linked to persistence of salmonellosis on pig farms. Feedstuffs 65, 11.

16. Nollet $\mathbf{N}$ et al. (2004) Risk factors for the herd-level bacteriologic prevalence of Salmonella in Belgian slaughter pigs. Preventive Veterinary Medicine 65, 63-75.

17. Erdman MM et al. (2005) Occurrence of Salmonella serotype Typhimurium DT104 on a commercial swine farm before, during, and after depopulation and repopulation. Journal of the American Veterinary Medical Association 227, 460-466.

18. van der Heijden M et al. (2005) Effectiveness of Salmonella control strategies in fattening pigs. In Proceedings of the 6th Symposium on the epidemiology and control of foodborne pathogens in pork, Rohnert park, California, USA.

19. van der Wolf PJ et al. (2001) Herd level husbandry factors associated with the serological Salmonella prevalence in finishing pig herds in The Netherlands. Veterinary Microbiology 78, 205-219.

20. Creus E et al. (2007) Effect of acidified feed on the prevalence of Salmonella in market-age pigs. Zoonoses and Public Health 54, 314-319.

21. Kranker S, Dahl J and Wingstrand A (2001) Bacteriological and serological examination and risk factor analysis of Salmonella occurrence in sow herds, including risk factors for high Salmonella seroprevalence in receiver finishing herds. Berliner Und Munchener Tierarztliche Wochenschrift 114, 350-352.

22. Papenbrock S et al. (2005) Investigations on prophylactic effects of coarse feed structure and/or potassium diformate on the microflora in the digestive tract of weaned piglets experimentally infected with Salmonella derby. Journal of Animal Physiology and Animal Nutrition 89, 84-87.

23. Berends BR et al. (1996) Identification and quantification of risk factors in animal management and transport regarding Salmonella spp. in pigs. International Journal of Food Microbiology 30, 37-53.

24. Aperce CC et al. (2010) Interaction of Bacillus species and Salmonella enterica serovar Typhimurium in immune or inflammatory signaling from swine intestinal epithelial cells. Journal of Animal Science 88, 1649-1656.

25. Letellier A et al. (2000) Assessment of various treatments to reduce carriage of Salmonella in swine. Canadian Journal of Veterinary Research 64, 27-31.

26. Beloeil PA et al. (2004) Risk factors for Salmonella enterica subsp. enterica shedding by market-age pigs in French farrow-to-finish herds. Preventive Veterinary Medicine 63, 103-120.

27. Smith RP et al. (2011) Investigating the association between gross pathological lesions and serological tests for Salmonella infection in pigs: an abattoir-based study. Veterinary Record 9, 168-240.

28. Gotter V et al. (2012) Main risk factors for Salmonella-infections in pigs in north-western Germany. Preventive Veterinary Medicine 106, 301-307.

29. Martelli F et al. (2018) Observations on the introduction and dissemination of Salmonella in three previously low prevalence status pig farms in the United Kingdom. Food Microbiology 71, 129-134.

30. Arnold ME et al. (2015) Evaluation of the sensitivity of faecal sampling for detection of monophasic Salmonella Typhimurium and other Salmonella in cattle and pigs. Epidemiology and Infection 143, 1681-1691.

31. Grimont PAD and Weill FX (2007) Antigenic Formulae of the Salmonella Serovars. 9th Edn. Paris, France: WHO Collaborating Centre for Reference and Research on Salmonella, Institut Pasteur.

32. Wilson JS et al. (2003) Nontyphoidal salmonellae in United Kingdom badgers: prevalence and spatial distribution. Applied and Environmental Microbiology 69, 4312-4315.

33. Fedorka-Cray PJ, Gray JT and Wray C Salmonella infections in pigs. In Wray C and Wray A (eds) Salmonella in Domestic Animals. Wallingford, UK: CABI Publishing, 2000, pp. 191-207.

34. Arnold ME and Cook AJC (2009) Estimation of sample sizes for pooled faecal sampling for detection of Salmonella in pigs. Epidemiology and Infection 137, 1734-1741.

35. Stark KDC et al. (2002) Differences and similarities among experts' opinions on Salmonella enterica dynamics in swine pre-harvest. Preventive Veterinary Medicine 53, 7-20. 